\title{
INTERthesis
}

DOUTORADO INTERDISCIPLINAR EM CIÊNCIAS HUMANAS - UFSC - FLORIANÓPOLIS - SC - BRASIL

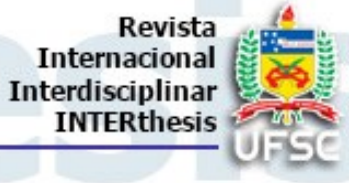

\section{BREVE HISTÓRIA DAS CLASSIFICAÇÕES EM PSIQUIATRIA ${ }^{1}$}

Fernanda Martinhago ${ }^{2}$

Sandra Caponi ${ }^{3}$

\section{Resumo:}

$\mathrm{Na}$ sociedade contemporânea há um grande número de pessoas diagnosticadas com transtornos mentais em diversos continentes. Diante deste contexto, busca-se delinear a trajetória histórica das classificações em psiquiatria, desde o século XIX até a atualidade. A primeira tentativa de classificações de patologias psiquiátricas foi em 1840 a partir da medição da frequência de duas categorias. A última classificação que antecede o primeiro Manual Diagnóstico e Estatístico de Transtornos Mentais (DSM) surge em 1918, com 22 categorias. No ano de 1952 é publicada a primeira edição do DSM, posteriormente, o Manual foi reformulado até a quinta edição, lançada em 2013. As classificações em psiquiatria foram criadas com a finalidade de obter dados estatísticos sobre a população e buscar uma linguagem universal sobre as patologias mentais. Na prática clínica há muitas controvérsias em relação as classificações em psiquiatria, pois esta rompe com as teorias de cunho crítico filosófico que constituíam as características das patologias mentais. A história nos mostra que houve uma fissura no modo de entender o sofrimento psíquico, e ali esvaíram-se a subjetividade e a história de vida dos sujeitos.

Palavras-chave: Psiquiatria. Classificação. Manual Diagnóstico e Estatístico de Transtornos Mentais.

\section{BRIEF HISTORY OF CLASSIFICATIONS IN PSYCHIATRY}

\begin{abstract}
:
In contemporary society, there is a large number of people diagnosed with mental disorders in several continents. In this context, we seek to delineate the historical trajectory of classifications in psychiatry, from the nineteenth century to the present day. The first attempt to classify psychiatric disorders was in 1840 from the frequency

\footnotetext{
${ }^{1} \mathrm{O}$ presente artigo resulta de uma parte da pesquisa realizada para a tese doutoral, desenvolvida de 2013 a 2017. Uma primeira versão do texto foi elaborada para o $15^{\circ}$ Seminário Nacional da História da Ciência e da Tecnologia, realizado em Florianópolis/SC, em 2016.

2 Pós-doutoranda do Programa de Pós-Graduação Interdisciplinar em Ciências Humanas da Universidade Federal de Santa Catarina, Florianópolis, SC, Brasil. Doutora em Antropologia e Comunicação pela Universitat Rovira i Virgili, Espanha, com mencão Doutorado Internacional Cum Laude. Estágio doutoral na Universidad de la República, Uruguay. Doutora em Ciências Humanas pela Universidade Federal de Santa Catarina, com sanduíche/cotutela na Universitat Rovira i Virgili, Itália. E-mail: martinhagofernanda@gmail.com

${ }^{3}$ Doutora em Lógica e Filosofia da Ciência pela Universidade de Campinas. Pós-doutorado na Universidade de Picardie, França, e Pós-doutorado Sênior na EHESS, Paris- França. Professora Titular do Departamento de Sociologia e Ciência Política da Universidade Federal de Santa Catarina, do Doutorado Interdisciplinar em Ciências Humanas, do Programa de Pós-Graduação em Sociologia Política, do Programa de Mestrado profissional em Saúde Mental da mesma Instituição, Florianópolis, SC, Brasil. E-mail: sandracaponi@gmail.com
} 
measurement of two categories. The last classification that precedes the first Diagnostic and Statistical Manual of Mental Disorders (DSM) appears in 1918, with 22 categories. In 1952, the first edition of the DSM was published, and later the Manual was reformulated until the fifth edition, which was launched in 2013. Classifications in psychiatry were created to obtain statistical data on the population and to seek a universal language on mental pathologies. In clinical practice, there are many controversies regarding the classifications in psychiatry since this breaks with the critical philosophical theories that constituted the characteristics of the mental pathologies. History shows us that there was a fissure in the way of understanding psychic suffering, and from there the subjectivity and the life history of the subjects were extinguished.

Keywords: Psychiatry. Classification. Diagnostic and Statistical Manual of Mental Disorders.

\section{BREVE HISTORIA DE LAS CLASIFICACIONES EN PSIQUIATRÍA}

\section{Resumen:}

En la sociedad contemporánea, hay un gran número de personas diagnosticadas con trastornos mentales en varios continentes. En este contexto, buscamos delinear la trayectoria histórica de las clasificaciones en psiquiatría, desde el siglo XIX hasta la actualidad. El primer intento de clasificar los trastornos psiquiátricos fue en 1840 a partir de la medición de frecuencia de dos categorías. La última clasificación que precede al primer Manual Diagnóstico y Estadístico de los Trastornos Mentales (DSM) aparece en 1918, con 22 categorías. En 1952, se publicó la primera edición del DSM, y luego se reformuló el Manual hasta la quinta edición, que se lanzó en 2013. Se crearon clasificaciones en psiquiatría para obtener datos estadísticos sobre la población y buscar un lenguaje universal sobre patologías mentales. En la práctica clínica existen muchas controversias con respecto a las clasificaciones en psiquiatría, ya que esto rompe con las teorías filosóficas críticas que constituyen las características de las patologías mentales. La historia nos muestra que hubo una fisura en la forma de entender el sufrimiento psíquico, y con esto se desvanecieron la subjetividad y la historia de vida de los sujetos.

Palabras clave: Psiquiatría. Clasificación. Manual Diagnóstico y Estadístico de los Trastornos Mentales.

\section{INTRODUÇÃO}

Na contemporaneidade há um número exorbitante de pessoas diagnosticadas com transtornos mentais em todas as partes do mundo. Segundo Whitaker (2011), a história contada para a sociedade é a de que a psiquiatria havia progredido no tratamento dos transtornos mentais, os pesquisadores estariam descobrindo as causas biológicas destes transtornos e a indústria farmacêutica havia desenvolvido medicamentos eficazes para o tratamento. Porém, ao fazer uma análise da incidência de transtornos mentais dos últimos 50 anos, Whitaker (2011) constatou que há uma verdadeira epidemia de transtornos mentais. Esta epidemia instiga 
pesquisadores de diversas áreas do conhecimento a investigarem como chegamos a tal fenômeno no século XXI. Assim, buscamos delinear a trajetória histórica das classificações em psiquiatria, do século XIX até a atualidade.

Desde a metade do século XIX, inicia-se uma nova forma de compreender e lidar com as doenças mentais. Os delírios, alucinações, atos violentos deixam de ser o foco da atenção e busca-se elaborar classificações de comportamentos e condutas que ocorrem na vida cotidiana dos sujeitos. Deste modo, vários comportamentos passam a ser considerados como desviantes e objeto de estudo e práticas médicas (CAPONI, 2012).

No período que corresponde a segunda metade do século XIX e início do século $\mathrm{XX}$, surgem as estratégias de intervenção e de gestão das populações relacionadas à teoria da degeneração de Morel, cuja proposta é criar uma classificação das doenças mentais. A ideia era "substituir uma classificação sintomática por uma classificação etiológica das doenças mentais, pois somente com a determinação das causas poderiam ser elaborados um sistema classificatório de patologias e uma terapêutica adequada" (CAPONI, 2012, p. 22).

Emil Kraepelin, no fim do século XIX e início do século XX, foi considerado o grande sistematizador da psicopatologia descritiva, quem consolidou a propensão nosológica. No período de 30 anos, houve oito edições do seu Manual de psiquiatria, sendo que todas apresentaram alterações nosológicas. Na perspectiva de Kraepelin, tratava-se de distinguir os diversos modos de sofrimento mental com base na clínica, que assim teriam o mesmo estatuto das doenças físicas que a medicina tratava (DUNKER, 2014).

Segundo Caponi (2012), desde a primeira edição (1883) até a oitava edição (1915) do Manual de Psiquiatria, o intuito de Kraepelin foi criar classificações de patologias psiquiátricas de modo que servissem como referência para a formação dos profissionais. As novas edições do Manual apresentavam ajustes, novos grupos de patologias, diagnósticos mais precisos, fundamentados nas descobertas científicas no campo da neurologia, das doenças cerebrais, da estatística médica, bem como estudos sobre herança.

Nos Estados Unidos, o principal objetivo para desenvolver uma classificação de transtornos mentais era obter informações estatísticas. A primeira tentativa foi em 1840 a partir da medição da frequência de duas categorias - idiotice e insanidade com a finalidade de constituir o Censo. Para o Censo do ano de 1880, haviam 
estabelecido sete categorias - mania, melancolia, monomania, paresia, demência, alcoolismo e epilepsia -, com o propósito de organizar o sistema asilar (APA, 2016). A última classificação que antecede o primeiro Manual Diagnóstico e Estatístico de Transtornos Mentais (conhecido como DSM, devido ao título original em inglês Diagnostic and Statistical Manual of Mental Disorders) surge em 1918, no Manual Estatístico para o Uso de Instituições de Insanos, com 22 categorias, entre elas, destacam-se a Psicose, Melancolia, Demência Precoce, Paranoia, Psiconeuroses e Neuroses (DUNKER, KYRILLOS NETO, 2011).

Até a Segunda Guerra Mundial, a psicanálise e a psiquiatria perpetuaram um sistema de trocas, o qual promoveu o progresso da psicopatologia, abarcou importações conceituais, criou campos de confluência metodológica, mutualismos diagnósticos, derivações semiológicas e hipóteses etiológicas (QUINET, 2001). No período entre os anos de 1900-1950, a psicanálise fundamentou a classificação norte-americana das doenças mentais, principalmente, com base nos conceitos de personalidade, estrutura e psicodinâmica (DUNKER, 2014).

\section{DSM I (1952)}

A partir da segunda metade do século $X X$, surgiu a necessidade de organizar sistemas diagnósticos, de modo que houvesse uma padronização nas categorias de doenças, as quais atenderiam as finalidades acadêmicas, terapêuticas, legais, administrativas e financeiras (ALARCÓN; FREEMAN, 2015). O intuito era estabelecer um consenso terminológico entre os clínicos, todavia não estava bem definido o limite entre o normal e o patológico (DUNKER, 2014). Com este propósito, em 1952, a Associação Americana de Psiquiatria (APA) publicou a primeira versão do DSM I com 106 categorias, pautado em um enfoque predominantemente psicanalítico. Nesse Manual, a reformulação psicopatológica estava baseada na "racionalidade diagnóstica centrada em tipos de reação e no pressuposto sintético da história de vida e das moções determinantes das doenças mentais" (DUNKER; KYRILLOS NETO, 2011, p. 6).

Adolf Meyer (1866-1950), psiquiatra suíço que foi presidente da APA, influenciou o sistema diagnóstico do DSM I, em que predominava categorias provenientes da psicodinâmica, salientando a oposição entre neurose e psicose. Era contrário à noção de processo e divisões propostas por Kraepelin. A racionalidade 
diagnóstica de Meyer estava voltada para os tipos de reação, levando em consideração a história de vida detalhada dos pacientes e as oscilações das doenças mentais. O primeiro grupo estava voltado, sobretudo, para a ansiedade até a depressão. Já o segundo grupo estava demarcado pelas alucinações e delírios somados à perda significativa da realidade. Ambos os grupos apresentavam aspectos que diziam respeito à etiologia biológica e condições responsivas a contextos sociais (DUNKER, 2014).

\section{DSM II (1968)}

O DSM II foi publicado em 1968, apresentando 182 categorias. Nesta edição, desaparece a noção de "reação" e há uma alteração terminológica originando a oposição entre neuroses e desordens de personalidade. Mantém-se o conceito de "neurose", o que demonstra o predomínio da psiquiatria psicodinâmica (DUNKER; KYRILLOS NETO, 2011). Esta versão do DSM não agradou a comunidade científica, pois a tentativa de confluir com a CID 8 (Classificação Internacional de Doenças) deixou a desejar em muitos aspectos, destaca-se a inclusão de 39 categorias a mais de transtornos mentais. Houve também discussões sobre a terminologia, principalmente, da esquizofrenia. Tais insatisfações levaram os especialistas a iniciarem uma nova revisão um ano depois de sua publicação (BARRIO, 2009).

\section{DSM III (1980)}

Em 1974, o psiquiatra Robert Spitzer comanda uma força tarefa com a finalidade de estabelecer uma nova versão do Manual. Um dos principais objetivos foi aperfeiçoar a uniformidade e a validade do diagnóstico psiquiátrico, bem como padronizar as práticas de diagnóstico entre os Estados Unidos e outros países. Esta proposta surgiu após os resultados de uma pesquisa que demonstrou diferenças significativas entre os diagnósticos psiquiátricos dos Estados Unidos e dos países europeus. A primeira iniciativa foi tornar a nomenclatura do DSM coerente com a Classificação Estatística Internacional de Doenças e problemas Relacionados à Saúde (DUNKER, 2014). 
No final da década de 1970, a confusão de línguas na psiquiatria era enorme, foi quando DSM III adotou os critérios da medicina baseada em evidências, recém surgidos na época. Esse fato foi considerado como uma revolução científica. A medicina baseada em evidências é a interface entre as pesquisas científicas e a prática clínica. As evidências científicas são utilizadas para orientar os diagnósticos e tratamentos na clínica (EL DIB, 2007). Segundo Campos; Onocko-Campos e Del Barrio , a perspectiva da medicina baseada em evidências preconiza uma hierarquia de evidências, em que umas esclareceriam mais a relidade que outras, dependendo do delineamento da pesquisa. Deste modo, os dados já estão estabelecidos a priori, independente de uma explicação da realidade. Algumas evidências tornam-se mais fortes, como as provenientes dos Ensaios Clínicos Randomizados (padrão ouro) e outras mais fracas nessa hierarquia, como as oriundas de estudos observacionais, experiências clínicas, entre outras. Neste contexto, a prática psiquiátrica é direcionada para identificação de sintomas, definição de diagnósticos e testar a eficácia dos medicamentos.

A ideia de uma classificação convencional, normativa e arbitrária de "transtornos mentais" foi bem aceita, uma vez que facilitaria o trabalho dos profissionais de saúde mental, das coberturas das empresas de seguro, pesquisa científica e da alocação dos recursos públicos em saúde mental (DUNKER, 2014). O objetivo foi a criação de uma classificação útil para tomar decisões e respaldar os tratamentos que incluíam categorias diagnósticas confiáveis, que fossem fruto do diálogo entre as diferentes abordagens da época, inclusive as psicossociais e a psicanalíticas (MARTÍNEZ-HERNÁEZ, 2000a). De modo geral, as vantagens do DSM III, foram a expansão e a melhoria na comunicação de diagnósticos (ALARCÓN; FREEMAN, 2015). Estabelecia-se assim a divisão bem definida do que eram os problemas do cotidiano e o que era de fato a doença mental. Segundo Martinez-Hernaez (2000a), o Manual foi elaborado em um contexto de biomedicalização da prática psiquiátrica, em que as classificações são obrigatoriamente distintas das teorias preconizadas pelas abordagens psicossociais e psicanalíticas dos manuais antecessores (DSM I e DSM II). A classificação feita no DSM III lembra as taxonomias desenvolvidas por Kraepelin entre 1885 e 1926 (MARTÍNEZ-HERNÁEZ, 2000b). O DSM III chega concomitantemente com a desinstitucionalização dos pacientes crônicos movida pela luta antimanicomial. A reformulação de políticas de saúde mental também foi baseada na ascensão dos 
tratamentos farmacológicos e na crítica à psicanálise por inspirar a prática diagnóstica da psiquiatria (DUNKER, 2014).

A publicação do DSM III ocorre em 1980, com 265 categorias, e apresenta uma posição ateórica, objetiva e supostamente neutra. Além disso, inseriu uma lógica de causalidade multifatorial, o que facilitou uma conexão com a epigenética (ALARCÓN; FREEMAN, 2015). As perspectivas psicodinâmicas e fisiológicas foram substituídas pelo método classificatório "multiaxial", sistema que busca uma forma mais adequada para o censo da população estatística, no lugar de um diagnóstico simples (DUNKER, 2014). Esta nova abordagem multiaxial e categórica apresenta critérios diagnósticos operacionalizados, guiados pela melhor confiabilidade e validade, ainda que não haja uma delimitação clara (ALARCÓN; FREEMAN, 2015).

$O$ termo neurose aparece pela última vez como categoria clínica. A eliminação do conceito de "neurose" gerou polêmica em função de que esta seria uma das classes fundamentais da psicopatologia psicanalítica e que justificaria a eficácia desse modo de psicoterapia. Os reformadores do DSM III consideraram que esse conceito tornara-se vago e não científico, o que pôs em risco a aprovação desta nova versão pelo Conselho de Administração da APA. Assim, estabeleceram um compromisso político de reutilização do termo, o qual foi mencionado entre parênteses, em alguns casos, depois da palavra "disorder". Portanto, pode-se considerar que as relações entre a psicanálise e a psiquiatria tiveram um ponto de virada demarcado pelo DSM III (DUNKER; KYRILLOS NETO, 2011; MALDONADO et al, 2011). Nesta edição, há a exclusão da categoria "homossexualidade" e a introdução do transtorno de personalidade narcisista (DUNKER; KYRILLOS NETO, 2011).

O DSM III é o marco da mudança de paradigma no âmbito da psiquiatria, que até o momento era regida, principalmente, com fundamentação na psicanálise. As patologias psiquiátricas passam a ser definidas por agrupamentos de sintomas, o que ocasionou a supressão das histórias de vida, das narrativas dos pacientes, das causas psicológicas e sociais que possivelmente causaram algum sofrimento psíquico e/ou sua manifestação em determinado comportamento (CAPONI, 2014). Esta transformação ocorre em função de um grupo de psiquiatras americanos, autodenominados neokraepelinianos e que, fundamentados nos novos avanços científicos, apresentavam estudos populacionais, bancos de dados quantitativos, 
descobertas da neurologia, anatomopatologia cerebral e da genética para elaborar o DSM III e as sucessivas edições do Manual (CAPONI, 2012).

Esta transformação na psiquiatria demarcada pelo DSM III, ao apresentar um sistema classificatório ateórico e operacional das síndromes psiquiátricas, modificou a concepção de pesquisa e da prática psiquiátrica. Esse manual disponibilizara à psiquiatria, sob a ótica descritivo-terminológico, um sistema preciso de diagnóstico e suporte para a pesquisa empírico-experimental. Os diagnósticos tornaram-se instrumentos convencionais, excluindo qualquer referência ontológica. Passou-se a exigir apenas a concordância no plano descritivo (DUNKER; KYRILLOS NETO, 2011). Com base em considerações descritivas, as classificações psicopatológicas passaram a ser definidas como síndromes, houve o abandono do enfoque psicodinâmico, prevaleceu o modelo biomédico para averiguar a diferença entre o normal e o anormal, e ainda foram acrescentadas muitas categorias novas de transtornos (MALDONADO et al, 2011).

Pela primeira vez, a psiquiatria, se posiciona em oposição à psicanálise, acusando os psicoterapeutas de criarem demandas e serviços para as pessoas que estavam apenas descontentes e não realmente doentes. Responsabilizava-os também pela superpopulação de internos nas instituições psiquiátricas (MAYES; HORWITZ, 2005). A psicanálise era um entrave para os psiquiatras que tinham uma visão fisicalista dos transtornos mentais, os quais estavam ligados à pesquisa experimental, e também para os psiquiatras progressistas, que acusavam a psicanálise de psicologizar problemas de ordem social (DUNKER; KYRILLOS NETO, 2011).

As transformações nos conceitos das enfermidades psiquiátricas no DSM III fazem com que os profissionais da saúde mental mudem os estilos de entrevistar, passando de um modo psicodinâmico, orientado pelo insight, para um modo descritivo, orientado pelo sintoma. Na entrevista orientada pelo sintoma parte-se do pressuposto que os distúrbios psiquiátricos se manifestam por meio de um conjunto peculiar de sinais e sintomas, um curso previsível e uma resposta a um tratamento específico. A meta neste tipo de entrevista é classificar as queixas e disfunções do paciente mediante as categorias determinadas pela classificação DSM. Assim, o diagnóstico indica um curso futuro e auxilia a selecionar o tratamento mais eficaz, porém não é possível um conhecimento sobre suas causas (OTHMER, 1992 apud DUNKER; KYRILLOS NETO, 2011). 
A versão revisada do DSM III, denominada DSM III-R, foi publicada em 1987 sob a direção de Spitzer, e apresentou 292 categorias. Esta versão recebeu diversas críticas por utilizar uma metodologia empobrecida para chegar aos critérios operacionais. As retificações dos conceitos foram consideradas prejudiciais, a etiologia como conceito central para identificar quadros mórbidos continuava evasiva, apresentava instabilidade diagnóstica, principalmente a curto prazo, e não havia marcadores biológicos que possibilitassem um avanço nosológico (ALARCÓN; FREEMAN, 2015).

\section{DSM IV (1994)}

O DSM IV foi publicado em 1994, com 297 categorias distribuídas em 886 páginas. O psiquiatra Allen Frances comandou um comitê de direção com 27 pessoas, as quais formaram 13 grupos de trabalho com 5 a 16 pessoas, sendo que cada grupo tinha um conselho composto em média por 20 pessoas. A principal alteração em relação às versões anteriores foi a inclusão de um critério de significância clínica para praticamente metade das categorias que tinham sintomas e causavam sofrimento clinicamente importante ou prejuízo no funcionamento social ou ocupacional, entre outras áreas (DUNKER, 2014). A histeria é desmembrada em síndromes: dissociação, disformismo corporal, ansiedade, depressão e fibromialgia. Este DSM exclui os psicodinamismos da etiologia conversiva e os substitui pelo enfoque neo-organicista, em contraposição ao organicismo anterior (DUNKER; KYRILLOS NETO, 2011).

Os diagnósticos psiquiátricos foram organizados no DSM IV em cinco eixos: Eixo I: distúrbios clínicos, incluindo os principais transtornos mentais, e desenvolvimento e distúrbios de aprendizagem; Eixo II: retardo subjacente penetrante ou condições de personalidade, bem como mental; Eixo III: situações clínicas agudas e doenças físicas; Eixo IV: fatores psicossociais e ambientais que contribuem para a desordem; e Eixo V: avaliação global de funcionamento.

Depois de aceitar o valor relativo de diversos fatores de validação, considerando resposta ao tratamento e prognóstico, o DSM IV revisou critérios diagnósticos de um moderado número de entidades, acrescentou e eliminou alguns transtornos, caracterizou subtipos de outros, incluiu em grupos afins, demarcou 
normas de interpretação e generalização das informações incluídas (ALARCÓN; FREEMAN, 2015).

Na versão atualizada deste Manual, no ano 2000, denominada de DSM-IVTR, aparecem mais 21 categorias no apêndice $B$, além das 297 já existentes na versão antiga. Nesta, há uma valorização de comorbidades e cruzamentos entre eixos diagnósticos (DUNKER; KYRILLOS NETO, 2011). A edição DSM-IV-TR não apresentou modificações substanciais, as críticas apontaram a falta de operacionalização dos diagnósticos e as retificações de muitas das entidades listadas. Observaram também dificuldades do uso em consultórios de Atenção Primária e centros de pesquisa, o que demandava a criação de um Manual específico para o uso nestas instituições. Outras críticas foram dirigidas ao seu uso imprudente e excessivo por advogados e administradores, com interpretações equivocadas, devido a partes do Manual consideradas ambíguas e imprecisas (ALARCÓN; FREEMAN, 2015).

Para Maldonado et al (2011), muitos critérios diagnósticos do DSM-IV-TR são extensos e difíceis de lembrar. Os autores consideram que a simplificação dos critérios diagnósticos poderia reduzir o seu uso inapropriado, além de ajudar o trabalho clínico dos psiquiatras, mesmo que, paradoxalmente, tenha que apresentar maior estrutura e rigidez.

Martínez-Hernáez (2000a) relata que nos novos DSMs se faz alusão à interpretação dos sintomas e dos transtornos mentais como não sendo uma tarefa própria da classificação, mas do clínico que fará um diagnóstico, de acordo com seu conhecimento e sua experiência. No entanto, a interpretação do que relata o paciente fica limitada à própria estrutura descritiva dos critérios diagnósticos já postos no DSM. Deste modo, torna-se mais difícil a compreensão e uma interpretação das expressões do paciente, uma vez que já haviam sido reconstruídas em um formato de manifestações biológicas.

\section{DSM-5 (2013)}

Durante a elaboração DSM-5, os trabalhos de campo foram posteriores à captação de uma diversidade de opiniões e comentários publicados por mais de 8.000 pesquisadores, clínicos, pacientes e familiares, além dos grupos de trabalhos designados. O enfoque principal foi a medição da gravidade dos sintomas e a avaliação em forma dimensional e transversal daqueles que apresentavam ampla 
diversidade de diagnóstico. Apesar do grande esforço para atingir os objetivos das tarefas propostas pelos organizadores, houve o apontamento de muitos erros nas estratégias para alcançar estes objetivos, como sigilo desnecessário, ambições de risco, métodos desorganizados e prazos irreais para o término (MALDONADO et al, 2011).

Esta última edição do DSM foi publicada em maio de 2013, com mais de 300 categorias, compondo 947 páginas, organizado em três sessões. A seção I apresenta as orientações para o uso clínico e forense. A seção II descreve os critérios e códigos diagnósticos dos transtornos. E por fim, na seção III estão os instrumentos para as avaliações dos sintomas, os critérios sobre a formulação cultural dos transtornos, o modelo alternativo para os transtornos de personalidade $\mathrm{e}$ uma descrição das condições clínicas para estudos posteriores (APA, 2013).

Assim como as demais versões, o DSM-5 está fundamentado no modelo categorial. Entretanto, sabendo das limitações deste sistema, seus autores incorporaram um enfoque mais dimensional em relação aos outros. Deste modo, é possível analisar se o sintoma é leve, moderado ou severo em diversos quadros clínicos. O sistema multiaxial foi suprimido porque gerava distinções superficiais e era pouco utilizado. Deste modo, as categorias diagnósticas dos eixos I e II do DSMIV-TR foram incluídas na seção II do DSM-5 com anotações separadas em cada categoria para as condições médicas associadas que pertenciam ao eixo III, para os fatores psicossociais e ambientais que caracterizavam o eixo IV e para incapacidades, ou seja, dificuldades no funcionamento social, laboral ou em outras áreas importantes da vida cotidiana que constituía o eixo $V$ do DSM-IV-TR. Na seção III, foram incluídas em todos os grupos diagnósticos, medidas dimensionais de severidade ou de frequência das últimas semanas em 13 conjuntos de sintomas, sendo 12 para crianças e adolescentes. Nesta edição do DSM, os capítulos foram organizados de modo que considerassem mais o ciclo de vida, em relação às edições anteriores. No início do Manual, estão os quadros clínicos que se manifestam nas primeiras fases do desenvolvimento, como os transtornos do desenvolvimento; na parte central, estão os transtornos que geralmente aparecem na adolescência e na idade adulta, como os transtornos de ansiedade, depressão ou do espectro da esquizofrenia; no final, estão os transtornos neurocognitivos, relacionados a velhice (ECHEBURÚA; SALABERRIA; CRUZ-SAEZ, 2014). 
DSM-5 traz uma lista de questões sociais que passam a ser consideradas como patologia, por exemplo: problemas de relacionamento, rompimentos familiares, negligência ou abuso parental, violência doméstica ou sexual, negligência ou abuso conjugal, problemas ocupacionais e profissionais, situações de falta de domicílio, problemas com vizinhos, pobreza extrema, baixo salário, discriminação social, problemas religiosos e espirituais, exposição a desastres, exposição a terrorismo e a não aderência ao tratamento médico. O que induz a exclusão da noção de sofrimento, somados à disseminação gerada pela recusa em pensar os sintomas no quadro como uma forma de vida (DUNKER, 2014).

Dunker (2014) nota que as renovações apresentadas no DSM-5 são questionadas por muitos psiquiatras por não se configurarem como descobertas científicas, mas redefinições dos nomes dos sintomas e definições operacionais de síndromes. Desta forma, o diagnóstico é sobrevalorizado mediante a análise retrospectiva dos efeitos de medicações em que o mecanismo de ação ainda é desconhecido (DUNKER, 2014).

Entretanto, para Alarcón e Freeman (2015), o DSM-5 apresentou diversos pronunciamentos ontológicos que enriqueceram o Manual: etiologia dos transtornos mentais fundamentadas em orientações neurobiológicas; confiabilidade e validade na classificações de modo equiparável ao que exige a medicina; avanços nas pesquisas básicas e clínicas, devido a muitos anos de trabalhos, com continuidade garantida de forma que possam ser atualizadas as novas versões do DSM, que está atualmente classificada com números arábicos DSM-5, justamente para ser seguida por DSM-5.1 e assim sucessivamente.

\section{CONSIDERAÇÕES FINAIS}

Desde o princípio as classificações psiquiátricas foram criadas com a finalidade de obter dados estatísticos sobre a população, bem como buscar uma linguagem universal sobre as patologias mentais de modo que suprisse as necessidades das organizações hospitalares, planos de saúde, atendimentos médicos e os indicadores para formulação de medicamentos.

Já para a prática clínica há muitas controvérsias em relação as classificações psiquiátricas, pois a psiquiatria rompe com as teorias de cunho crítico filosófico que constituíam as características das patologias mentais. Tais influências filosóficas 
podem ser observadas em Pinel que se baseia no pensamento Hegel, e em Kraepelin (psiquiatria clássica alemã) pela teoria de Kant, em Esquirol e Morel (psiquiatria clássica francesa) se fundamenta no positivismo contemporâneo e na psiquiatria de Karl Jaspers influenciada por Husserl (BERRIOS, 1996). A partir do DSM III, a psiquiatria dá uma grande virada e passa a se considerar como ateórica, organizando-se exclusivamente a partir de sintomas que devem ser quantificados num periodo determinado de tempo, desestimando qualquer referencia à psicanalise, modelo que persistirá nas posteriores edições do Manual até o DSM-5 (CAPONI, 2012; MARTINHAGO, 2017). O período de tempo em que aparecem os sinais e sintomas, considerado para realizar os diagnósticos dos diferentes transtornos, também varia de acordo às diferentes edições do DSM. Assim, quando pensamos no luto, podemos observar que no DSM-IV eram considerados como caracterizando um episódio depresivo, os sintomas estão presentes por um período de dois meses, enquanto que no DSM-5 o período de presença dos sintomas indesejados se reduz a duas semanas. Evidencia-se assim, a incapacidade de este Manual para entender que os sofrimentos perante uma perda ou fracaso, são próprios da condição humana e não podem ser simplesmente reduzidos a um diagnóstico que se limite a observar a presença de sintomas em um período de tempo pré-determinado.

Pouco antes da publicação do DSM-III, ao longo dos anos 1970, assistimos à Reforma Psiquiátrica italiana, modelo para a Reforma Psiquiátrica brasileira, e as críticas de um conjunto de intelectuais como Foucault, Goffmann, Thomas Szasz, Basaglia, Laing, dentre outros. Críticas embasadas no direito ao tratamento em liberdade, apostando na reinserção social, no resgate à cidadania dos sujeitos, implementação de políticas públicas sob a forma de lei, portarias e outras regulações para que fossem criados serviços de atenção à saúde mental (CAMPOS; ONOCKO-CAMPOS; DEL BARRIO, 2013). Ambas tiveram como objetivo desenvolver práticas desinstitucionalizantes e desmedicalizantes, em que a atenção se voltasse para o sujeito e não para a doença. A desinstitucionalização reside na desconstrução do paradigma problema-solução que significa, no âmbito da psiquiatria, o diagnóstico como definidor do sujeito (MARTINHAGO; OLIVEIRA, 2015). Basaglia (1985), precursor da Reforma Psiquiátrica italiana, propôs colocar a doença entre parênteses e olhar para o sujeito. Estas Reformas promoveram uma transformação no que diz respeito a atenção à saúde mental, surgindo assim, 
diversas propostas de inovação na prática clínica para esse público, como a clínica peripatética e a clínica ampliada.

A clínica peripatética proposta por Lancetti (2008), se caracteriza como uma ferramenta para entender as experiências clínicas que ocorrem em movimento, fora dos consultórios, nos espaços que transbordam a psiquiatria, a psicanálise e as instituições de saúde mental, que emergem conforme a complexidade do tratamento. O autor utiliza o termo peripatético no sentido de passear, ir e vir conversando.

A clínica ampliada proposta por Campos e Amaral (2007) amplia o objeto de trabalho da clínica, de modo que não fique restrito à doença, mas englobe também situações que aumentam a vulnerabilidade das pessoas, ou seja, uma "clínica do sujeito". Nesta perspectiva, a finalidade do trabalho clínico ultrapassa a busca pela saúde por meios curativos, preventivos, de reabilitação, propondo ampliar a autonomia dos sujeitos, fortalecendo sua capacidade de lidar com sua rede ou sistema de dependências, formada pelo contexto social e cultural, sua própria subjetividade e das relações de afetos que estão entrelaçados.

Podemos afirmar que, em resposta a essas demandas e reivindicações a psiquitria optou por reforçar ainda mais sua posição reducionista clássica, aquela que predominou ao longo do século $\mathrm{XIX}$ e inicios do século $\mathrm{XX}$ na psiquaitria biológica. As críticas levantadas pelos teóricos e psiquiatras de fins dos anos 1970, caminhavam em sentidos opostos ao proposto pelo DSM-III, cujo enfoque está centrado nos sintomas. Esses intelectuais,

Somados às criticas dos movimentos pelos direitos, reclamavam uma resposta do campo da psiquiatria. Porém, essa resposta chegou do modo mais inesperado. Longe de uma reflexão crítica dos postulados questionados pelos defensores de direitos, ocorreu uma radicalização e um retorno às velhas teses da psicologia biológica do século XIX. Nesse momento e após longos debates será publicado o DSM-III, inaugurando um novo modo de se entender a psiquiatria (CAPONI, 2012, p. 163/164).

Mediante o contexto histórico apresentado podemos observar que as alternativas mais democráticas, como a clínica peripatética (LANCETTI, 2008) e a clínica ampliada (CAMPOS; AMARAL, 2007), escapam as classificações psiquiátricas atuais.

A história das classificações em psiquiatria, nos mostra que, nesta perspectiva, houve uma fissura no modo de entender o sofrimento psíquico, e ali esvaíram-se a subjetividade e a história de vida dos sujeitos. Nesta direção, passou- 
se a considerar o ser humano como corpos que manifestam comportamentos ditos desviantes e precisam de intervenções medicamentosas.

\section{Agradecimentos}

À Fundação de Amparo à Pesquisa e Inovação do Estado de Santa Catarina (FAPESC) e à Coordenação de Aperfeiçoamento de Pessoal de Nível Superior (CAPES) pelas bolsas de doutorado no Brasil e no exterior que possibilitaram a realização da pesquisa. 


\section{REFERÊNCIAS}

ALARCON G, Renato D.; FREEMAN, A. M. Rutas ontológicas de la nosología psiquiátrica: ¿Cómo se llegó al DSM-5?. Rev Neuropsiquiatr, Lima, v. 78, nº 1, enero 2015.

APA - American Psychiatric Association. DSM History. Disponível em: https://www.psychiatry.org/psychiatrists/practice/dsm/history-of-the-dsm Acesso em 18/08/2016.

BARRIO, V. Raíces y evolución del DSM. Revista de historia de la psicología, Valencia, vol. 30, $n^{\circ}$ 2-3, p. 81-90, 2009.

BERRIOS, G. The History of Mental Symptoms: Descriptive Psychopathology Since the Nineteenth Century. Cambridge: Cambridge University Press, 1996.

BASAGLIA, F. A instituição negada: relato de um hospital psiquiátrico. 2. ed. Rio de Janeiro: Graal, 1985.

CAMPOS, G. W. de S.; ONOCKO-CAMPOS, R. T.; DEL BARRIO, L. R. Políticas e práticas em saúde mental: as evidências em questão. Ciênc. saúde coletiva [online]. 2013, vol.18, n.10, pp.2797-2805.

CAMPOS, G. W. de S.; AMARAL, M. A. do. A clínica ampliada e compartilhada, a gestão democrática e redes de atenção como referenciais teórico-operacionais para a reforma do hospital. Ciênc. saúde coletiva [online]. 2007, vol.12, n.4, pp.849-859.

CAPONI, S. O DSM-V como dispositivo de segurança. Physis, Rio de Janeiro, v. 24, $\mathrm{n}^{\circ} 3$, p. 741-763, Sept. 2014.

CAPONI, S. Loucos e Degenerados: uma genealogia da psiquiatria ampliada. Rio de Janeiro: FIOCRUZ, 2012.

DUNKER, C. I. L.; KYRILLOS NETO, F. A psicopatologia no limiar entre psicanálise e a psiquiatria: estudo comparativo sobre o DSM. Vínculo, São Paulo, v. 8, n. 2, p. 1-15, dez. 2011.

DUNKER, C. I. L. Questões entre a psicanálise e o DSM. J. psicanal., São Paulo , v. 47, n. 87, p. 79-107, dez. 2014. 
ECHEBURÚA, E.; SALABERRIA, K.; CRUZ-SAEZ, M. Aportaciones y Limitaciones del DSM-5 desde la Psicología Clínica. Ter Psicol [online]. Santiago, vol.32, n.1, p. 65-74, 2014.

EL DIBE, R. P. Como praticar a medicina baseada em evidências. J Vasc Bras, Vol. $6, \mathrm{~N}^{\circ} 1,2007$.

LANCETTI, A. Clínica Peripatética. São Paulo: Hucitec, 2008.

MAYES, R.; HORWITZ, A. DSM-III and the revolution in the classification of mental illness. J Hist Behav Sci, New Jersey, n. 41, v. 3, p. 249-67, 2005.

MALDONADO, G. et al . EI DSM-V. Luces y sombras de un manual no publicado. Retos y expectativas para el futuro. Salud Ment, México , v. 34, n. 4, p. 367-378, agosto 2011.

MARTÍNEZ-HERNÁEZ, A. What's Behind The Symptom? On Psychiatric Observation and Anthropological Understanding. New York: Taylor \& Francis, 2000a.

MARTÍNEZ-HERNÁEZ, A. Anatomía de una ilusión. EI DSM-IV y la biologización de la cultura. In: PERDIGUERO, E. COMELLES, J.M. (Org). Medicina y Cultura: Estudios entre la antropología y la medicina. Barcelona: Edicions Bellaterra, 2000b, p. 249-275.

MARTINHAGO, Fernanda. Contágio social de transtornos mentais: análise das estratégias biopolíticas de medicalização da infância. 300 p. Tese (Doutorado) Universidade Federal de Santa Catarina, Centro de Filosofia e Ciências Humanas, Programa de Pós-Graduação Interdisciplinar em Ciências Humanas, Florianópolis, 2017.

MARTINHAGO, F.; OLIVEIRA, W. F. (Des)institucionalização: a percepção dos profissionais dos Centros de Atenção Psicossocial de Santa Catarina, Brasil. Saúde e Sociedade (Online). São Paulo, v.24, p.1273-1284, 2015.

QUINET, A. Psicanálise e psiquiatria: controvérsias e convergências. Rio de Janeiro: Rios Ambiciosos, 2001. 
WHITAKER, R. Anatomía de una epidemia: medicamentos psiquiátricos y el asombroso aumento de las enfermedades mentales, Trad. J. Manuel Álvarez. Madrid: Capitán Swing, 2011. 